\title{
Avaliação do gerenciamento de desperdícios e das condições higiênico-sanitárias de pizzaria localizada no norte da Bahia
}

\author{
Evaluation of waste management and hygienic-sanitary conditions of a pizzeria located in the north \\ of Bahia
}

Evaluación de manejo de residuos y condiciones higiénico-sanitarias de una pizzería ubicada en el norte de Bahía

Recebido: 23/04/2021 | Revisado: 01/05/2021 | Aceito: 20/05/2021 | Publicado: 08/06/2021

\author{
Antonio Alves dos Santos \\ ORCID: https://orcid.org/0000-0002-2800-2759 \\ Universidade de Pernambuco, Brasil \\ E-mail: antonio.alvessantos@upe.br \\ Virgínia Cabral Benício \\ ORCID: https://orcid.org/0000-0002-5451-3499 \\ Universidade de Pernambuco, Brasil \\ E-mail: vivibenicio18@hotmail.com \\ Jeane de Souza Silva \\ ORCID: https://orcid.org/0000-0002-9901-2727 \\ Universidade de Pernambuco, Brasil \\ E-mail: jeane.souza@upe.br \\ Joelma Barbosa da Silva \\ ORCID: https://orcid.org/0000-0002-1009-7761 \\ Universidade de Pernambuco, Brasil \\ E-mail: bjoelma6.jb@gmail.com \\ Ágatha Cristhine de Souza Alencar Lima \\ ORCID: https://orcid.org/0000-0002-6748-2716 \\ Universidade de Pernambuco, Brasil \\ E-mail: cristhineagatha@gmail.com \\ Janiclecia Macedo Albuquerque \\ ORCID: https://orcid.org/0000-0003-4543-6767 \\ Universidade de Pernambuco, Brasil \\ E-mail: janiclecia05@gmail.com \\ Ana Caroline Januario Filipe \\ ORCID: https://orcid.org/0000-0003-3320-7297 \\ Universidade de Pernambuco, Brasil \\ E-mail: anacarolfilipe@gmail.com \\ Maria Vanessa Feitoza da Silva \\ ORCID: https://orcid.org/0000-0002-7842-5485 \\ Universidade de Pernambuco, Brasil \\ E-mail: mariavanessamv16@gmail.com \\ Claudileide de Sá Silva \\ ORCID: https://orcid.org/0000-0002-3677-3000 \\ Universidade de Pernambuco, Brasil \\ E-mail: claudileide.silva@upe.br
}

\begin{abstract}
Resumo
O desperdício é uma das principais causas do aumento dos custos na produção de refeições. Assim, objetivou-se avaliar o gerenciamento de desperdícios e as condições higiênico-sanitárias e microbiológicas de alimentos e superfícies de uma pizzaria no norte da Bahia. Trata-se de um estudo descritivo transversal, qualitativo e quantitativo. O diagnóstico higiênico-sanitário foi realizado através da aplicação de checklist conforme a RDC n 216/2004, ANVISA, e a análise microbiológica realizada para a contagem de coliformes totais, Escherichia coli, Staphylococcus aureus e presença/ausência de Salmonella, nas amostras de alimentos. Para análise de superfícies e equipamentos foi utilizada a técnica de swabs e a contagem de coliformes totais e Escherichia coli através do sistema de análise Petrifilm Coliform Count Plate $3 \mathrm{M} ®$. Observou-se valores aceitáveis de coliformes totais na tábua de pizza, tomate e frango, com valores de $1,40 \mathrm{x}$ $10^{3} \mathrm{UFC} / \mathrm{cm}^{2}, 1,04 \times 10^{3} \mathrm{UFC} / \mathrm{g}, 1,70 \times 10^{3} \mathrm{UFC} / \mathrm{g}$, respectivamente; e inaceitáveis no processador de alimentos, correspondente a 4,88 x $10^{3} \mathrm{UFC} / \mathrm{cm}^{2}$. Encontrou-se $1,3 \times 10^{3} \mathrm{UFC} / \mathrm{mL}$, coliformes totais na água, onde deveria estar ausente. Não foram encontradas colônias de Escherichia coli nem presença de Salmonella nas amostras. Quanto às condições higiênico-sanitárias, a pizzaria foi classificada no grupo 3 (0 a $50 \%$ de atendimento dos itens), segundo a RDC n 275/2002. Logo, a presença de um responsável técnico em
\end{abstract}


serviço de alimentação é necessária, apresentando uma relação direta no controle de desperdícios e higiênico-sanitário, promovendo ações corretivas que proporcionam a diminuição dos riscos associados à contaminação dos alimentos e otimização dos custos operacionais.

Palavras-chave: Boas práticas; Restaurante; Coliformes totais; Escherichia coli; Staphylococcus aureus; Desperdício.

\begin{abstract}
Waste is one of the main causes of increased costs in the production of meals. Thus, the objective was to evaluate waste management and the hygienic-sanitary and microbiological conditions of food and surfaces in a pizzeria in northern Bahia. This is a cross-sectional, qualitative and quantitative study. The hygienicsanitary diagnosis was carried out through the application of a checklist according to RDC $\mathrm{n}^{\circ} 216 / 2004$, ANVISA, and the microbiological analysis performed for the counting of total coliforms, Escherichia coli, Staphylococcus aureus and the presence / absence of Salmonella, in the food samples . For the analysis of surfaces and equipment, the swab technique and the counting of total coliforms and Escherichia coli were used through the Petrifilm Coliform Count Plate $3 \mathrm{M} \circledast$ analysis system. Acceptable values of total coliforms were observed on the pizza, tomato and chicken board, with values of $1.40 \times 10^{3} \mathrm{UFC} / \mathrm{cm}^{2}, 1.04 \times 10^{3} \mathrm{UFC}$ $/ \mathrm{g}, 1.70 \times 10^{3} \mathrm{UFC} / \mathrm{g}$, respectively; and unacceptable in the food processor, corresponding to $4.88 \times 10^{3}$ UFC / $\mathrm{cm}^{2}$. It was found $1.3 \times 10^{3} \mathrm{UFC} / \mathrm{mL}$, total coliforms in the water, where it should be absent. There were no Escherichia coli colonies or Salmonella in the samples. As for the hygienic-sanitary conditions, the pizzeria was classified in group 3 ( 0 to $50 \%$ of the items' service), according to RDC $n^{\circ} 275 / 2002$. Therefore, the presence of a technician in charge of food service is necessary, presenting a direct relationship in the control of waste and hygienic-sanitary, promoting corrective actions that provide the reduction of risks associated with food contamination and optimization of operating costs.
\end{abstract}

Keywords: Good practices; Restaurant; Total coliforms; Escherichia coli; Staphylococcus aureus; Waste.

\title{
Resumen
}

El desperdicio es una de las principales causas del aumento de costos en la producción de comidas. Así, el objetivo fue evaluar la gestión de residuos y las condiciones higiénico-sanitarias y microbiológicas de alimentos y superficies en una pizzería del norte de Bahía. Se trata de un estudio transversal, cualitativo y cuantitativo. El diagnóstico higiénico-sanitario se realizó mediante la aplicación de un checklist según RDC $\mathrm{n}^{\mathrm{o}}$ 216/2004, ANVISA, y el análisis microbiológico realizado para el conteo de coliformes totales, Escherichia coli, Staphylococcus aureus y la presencia / ausencia de Salmonella, en las muestras de comida. Para el análisis de superficies y equipos se utilizó la técnica del hisopo y el conteo de coliformes totales y Escherichia coli a través del sistema de análisis Petrifilm Coliform Count Plate $3 \mathrm{M} \AA$. Se observaron valores aceptables de coliformes totales en la tabla de pizza, tomate y pollo, con valores de $1,40 \times 10^{3} \mathrm{UFC} / \mathrm{cm}^{2}$, $1,04 \times 10^{3} \mathrm{UFC} / \mathrm{g}, 1,70 \times 10^{3} \mathrm{UFC} / \mathrm{g}$, respectivamente; e inaceptable en el procesador de alimentos, correspondiente a 4,88 x $10^{3} \mathrm{UFC} / \mathrm{cm}^{2}$. Se encontró $1,3 \times 10^{3} \mathrm{UFC} / \mathrm{mL}$, coliformes totales en el agua, donde debería estar ausente. No hubo colonias de Escherichia coli o Salmonella en las muestras. En cuanto a las condiciones higiénico-sanitarias, la pizzería se clasificó en el grupo 3 (0 a 50\% del servicio de los artículos), según RDC n ${ }^{\circ} 275 / 2002$. Por ello, es necesaria la presencia de un técnico encargado del servicio de alimentación, presentando una relación directa en el control de residuos e higiénico-sanitario, promoviendo acciones correctivas que brinden la reducción de riesgos asociados a la contaminación de alimentos y optimización de costos operativos.

Palabras clave: Buenas prácticas; Restaurante; Coliformes totales; Escherichia coli; Staphylococcus aureus; Desperdicio.

\section{Introdução}

$\mathrm{Na}$ atualidade a rotina das pessoas vem sendo modificada devido às alterações das cargas horárias de trabalho, tempo gasto na locomoção, crescimento dos centros urbanos; e isso reflete diretamente nos hábitos alimentares da população, que por sua vez deixam de comer em suas residências e optam por serviços de alimentação que oferecem praticidade diante da imensa movimentação do dia a dia (Marques et al., 2017). É notável o crescimento do setor alimentício nos últimos anos, em especial, o mercado de pizzarias; movimentando cerca de 22 bilhões de reais por ano (Silva, 2019). Neste sentido, também vem crescendo cada vez mais, os serviços de delivery, que associado ao atual momento de distanciamento físico, devido ao COVID-19, tornou-se indispensável (Botelho, 2020).

Com esse crescimento da alimentação fora de casa, aliado a adesão dos serviços delivery, aumenta também o risco de contaminação através dos alimentos ingeridos, tendo em vista que nem todos os estabelecimentos se prestam a fornecer 
produtos de qualidade do ponto de vista higiênico sanitário (Ponath et al., 2016).

A contaminação dos alimentos pode surgir desde a etapa da colheita da matéria prima, percorrendo o processamento, até a sua distribuição, sendo a causa de Doenças Transmitidas por Alimentos (DTAs) (Veiga, Antonacio \& Belmonte, 2020). Pode-se citar como uma potencial fonte de contaminação, as práticas de manipulação inadequada do alimento, além da forma de armazenamento, temperatura e tempo de cocção, exposição ao consumo, à qualidade da matéria prima e higienização dos utensílios (Guimarães, Ferreira \& Soares, 2018).

A falta de treinamento da equipe envolvida em unidades de alimentação (UAN) vai além da possibilidade de contaminação dos alimentos, podendo acarretar custos relacionados a desperdícios, que por sua vez é um fator indicativo de qualidade de serviço prestado (Abreu, Spinelli \& Pinto, 2003). Diferentemente do que se costuma pensar, não há desperdícios apenas relacionados a alimentos, mas tudo que envolve custos como mão de obra, planejamento de cardápio, dimensionamento de estoque, eletricidade, o porcionamento das preparações e outros (Alves, Rios \& Matos, 2020).

Os serviços de alimentação devem ter como objetivo garantir um planejamento eficiente e eficaz de todo o processo referente à manipulação, distribuição e melhor aproveitamento dos alimentos, assegurando tanto a redução de custos, como a segurança alimentar do ponto de vista microbiológico (Parisoto, Hautrive \& Cembranel, 2013). Pois, sabe-se que os comensais têm se tornado mais exigentes e buscam por serviços que lhes proporcionem não somente um alimento seguro, mas também a satisfação das suas necessidades e desejos, tanto nos aspectos sensoriais quanto nutricionais das preparações (Viana \& Ferreira, 2017).

Tendo em vista a relevância do impacto da contaminação alimentícia na saúde humana, esse estudo teve como finalidade avaliar o perfil higiênico-sanitário, analisando a presença de microrganismos contaminantes (coliformes totais, Escherichia coli, Staphylococcus aureus, e Salmonella), nos utensílios, alimentos, equipamentos e água, assim como verificar o gerenciamento dos desperdícios em uma pizzaria localizada na cidade de Juazeiro, Estado da Bahia, Brasil.

\section{Metodologia}

Trata-se de um estudo de caráter descritivo transversal e quantitativo, seguindo a metodologia científica descrita por Pereira et al. (2018), realizada em Março de 2021. Para tanto, ocorreu uma visita técnica em uma pizzaria, na cidade de Juazeiro, Sertão do São Francisco, Bahia, Brasil. O estabelecimento sob estudo possui em seu quadro, 6 funcionários durante a semana e 8 aos finais de semana, devido a maior demanda de serviços. Em média, sua produção durante a semana é de 80 unidades de pizzas; já aos finais de semana, a demanda pode chegar a 120 ou mais. A empresa analisada é gerenciada pelo proprietário, que por sua vez, não possui formação acadêmica e conta apenas com seus conhecimentos adquiridos em um curso de pizzaiolo.

As análises microbiológicas foram realizadas para verificação e contagem de coliformes totais, Escherichia coli, Salmonella e Staphylococcus aureus em amostras de alimentos, através do sistema Petrifilm 3M®, e classificadas de acordo com a IN n60/2019. Através da técnica de Swab, foram coletadas amostras de equipamentos e superfícies, sendo assim realizada na superfície interna do processador, como também na tábua para pizza, a qual entra em contato direto com o alimento pronto para consumo. A classificação da adequação das superfícies, foi realizada de acordo com o descrito por Freitas et al. (2020) que preconiza o limite de colônias de coliformes totais de até 2 por $\mathrm{cm}^{2}$.

Para realização do diagnóstico das condições higiênico-sanitárias, foi utilizada uma lista de verificação contendo 174 itens, construído a partir da RDC n 216/2004, da Agência Nacional de Vigilância Sanitária (ANVISA), e CVS 5/2013, do Centro de Vigilância Sanitária de São Paulo. Itens desta lista foram distribuídos nas seguintes seções: I) Edificação e instalações; II) Higienização; III) Controle integrado de vetores de pragas; IV) Abastecimento de água V) Manejo de resíduos; VI) Manipuladores VII) Matérias primas, ingredientes e embalagens; VIII) Preparação do alimento; IX) Armazenamento e 
transporte dos alimentos preparados; X) Exposição ao consumo; XI) Documentação e registro; XII) Responsabilidade. Para classificação do estabelecimento segundo o percentual de adequação, foi utilizada a RDC no 275/2002 da ANVISA, que classifica o estabelecimento em: Grupo 1 (76 a 100\% de adequações), Grupo 2 (51 a 75\% de adequações) e Grupo 3 (0 a 50\% de adequações).

Para avaliar o gerenciamento do desperdício, foi construída e aplicada uma lista de verificação com base nos itens descritos, composta por 85 itens no total e 16 tópicos, sendo eles: I) Cardápio; II) Compras; III) Recebimento; IV) Armazenamento; V) Pré-preparo; VI) Preparo; VII) Porcionamento e distribuição; VIII) Fator de correção; IX) Sobras e restos, X) Mão de obra; XI) Tempo; XII) Materiais de higiene; XIII) Materiais de escritório; XIV) Descartáveis; XV) Eletricidade; XVI) Água. Para realização do cálculo do percentual de conformidade dos checklists, o total de itens considerados conformes foi multiplicado por 100 e o resultado foi dividido pelo total de itens presentes em cada tópico, desconsiderando os que não se aplicam ao estabelecimento analisado.

O banco de dados foi construído no Excel, versão 7.0, para Windows 10, e as análises estatísticas realizadas no software Prism, versão 5.0 (GraphPad, USA). Para tanto, utilizou-se de estatística descritiva com diferenças com valor de $p$ $<0,05$, sendo considerados estatisticamente significantes.

\section{Resultados e Discussão}

\section{Análises microbiológicas}

A partir da análise microbiológica, foi possível verificar que das cinco amostras coletadas, três encontraram-se conformes, segundo os parâmetros estabelecidos pela Agência Nacional de Vigilância Sanitária, instrução normativa ${ }^{\circ}$ 60/2019.

Nesse estudo, todas as amostras analisadas apresentaram-se com a contagem de Staphylococcus aureus ausente ou incapaz de se manifestar. Segundo a legislação, a presença de Staphylococcus aureus não deve ultrapassar 104 UFC/g em alimento; devendo estar ausente em água e superfícies (ANVISA IN nº6/2019).

Ao avaliar a contagem de coliformes totais, a qual utilizou neste estudo com intuito de quantificar possíveis enterobactérias, a água e o processador de alimentos apresentaram-se inadequados, indicando a presença de coliformes totais, conforme pode ser observado na tabela 1, e assim não cumprindo com as normas descritas pela Portaria n 2.914/2011 e Freitas et al. (2020), respectivamente. Também não foram encontradas cepas de Escherichia coli e Salmonella nas amostras de alimentos, assim como, não foram encontradas cepas de Escherichia coli em nenhuma das superfícies analisadas. Neste sentido, a classificação quanto à contagem de coliformes totais não deve ultrapassar o valor de $\leq 100 \mathrm{UFC} / \mathrm{cm}^{2}$ em superfícies, devendo estar ausente em água, sendo analisadas conforme descrito por Freitas et al. (2020) e Portaria n ${ }^{\circ}$ 2.914/2011. 
Tabela 1. Análises microbiológicas de superfície e alimentos de uma empresa prestadora de serviços em alimentação em uma cidade do norte baiano, 2021.

\begin{tabular}{llc}
\hline ITENS & S. aureus & Coliformes totais \\
Processador de alimentos & - & $4,88 \times 10^{3}{\mathrm{UFC} / \mathrm{cm}^{2}}^{2}$ \\
Tábua de pizza & - & $1,40 \times 10^{3} \mathrm{UFC} / \mathrm{cm}^{2}$ \\
Tomate & Ausente & $1,04 \times 10^{3} \mathrm{UFC} / \mathrm{g}$ \\
Frango & - & $1,70 \times 10^{3} \mathrm{UFC} / \mathrm{g}$ \\
Água & - & $1,3 \times 10^{3} \mathrm{UFC} / \mathrm{mL}$ \\
\hline
\end{tabular}

Fonte: Autores.

Diante dos resultados observados neste estudo, percebe-se que todas as amostras de alimentos coletadas se enquadram em um perfil satisfatório de segurança microbiológica, pois segundo a contagem dos microrganismos presentes, apresentaramse com valores que não indicam riscos à saúde do comensal (Monte et al., 2021).

Assim como nos gêneros alimentícios, é imprescindível garantir a segurança higiênico-sanitária das superfícies de utensílios e equipamentos, pois caso não haja essa garantia, os alimentos acabam se tornando inseguros por estarem com seu padrão microbiológico comprometido (Santos et al., 2020).

De acordo com a Portaria no 326/1997 os utensílios e equipamentos usados em estabelecimentos que comercializam alimentos, não podem constituir risco para a saúde e nem devem comprometer a higiene dos alimentos ou serem fontes de contaminação. Neste contexto, ao avaliar as superfícies, constatou-se uma quantidade elevada de coliformes totais no processador de alimentos. Os valores encontrados são inadequados, conforme descrito por Freitas et al. (2020). Possivelmente, os resultados do presente estudo associam-se à falhas na higienização desses equipamentos, podendo levar à contaminação cruzada no processo de fabricação e pós-produção do alimento (Rezende et al., 2012). Os valores obtidos nesta pesquisa também são semelhantes aos descritos por Chaves et al. (2014), os quais avaliaram uma Unidade de Alimentação e Nutrição em São Luís - MA, sendo constatado que dos equipamentos e utensílios analisados, alguns estavam contaminados com coliformes totais.

Já em um estudo realizado por Guimarães, Ferreira e Soares (2018), avaliou-se as condições microbiológicas de utensílios utilizados no processo de produção de alimentos em duas Unidades de Alimentação e Nutrição (UANs) do estado da Bahia, sendo constatado que dos 5 utensílios comuns em cada unidade, todos apresentaram algum tipo de contaminação microbiana; além disso, as análises microbiológicas demonstraram que a contaminação mais frequente foi por mesófilos e estafilococos, sendo somente três utensílios contaminados por coliformes totais e Escherichia coli, mostrando portanto, um menor índice de contaminação de origem fecal. Com isso, acredita-se que a realização de treinamento dos manipuladores relacionada à correta higienização das superfícies dos equipamentos e utensílios poderia solucionar este tipo de contaminação (Souza \& Campos, 2003).

Em relação à água, sabe-se que essa chega ao estabelecimento em questão a partir da empresa de abastecimento público da cidade. E a presença de microrganismos na água pode resultar em surtos e Doenças Transmitidas por Alimentos (DTAs), sendo responsável por sérios problemas de saúde pública (Almeida et al., 2009; Oliveira et al., 2010). Estes resultados podem refletir a contaminação das redes de tubulação ou do reservatório de água do estabelecimento. Resultados semelhantes também foram relatados por Silva et al. (2013), os quais avaliaram a qualidade microbiológica da água utilizada em UANs no 
município de Caruaru- PE, onde coletou 20 amostras de água para verificação da presença de coliformes totais e termotolerantes; e entre as análises realizadas, 6 foram confirmatórias para presença desses. Visto isso, segundo a Portaria $\mathrm{n}^{\circ}$ 2.914/2011, a água utilizada para consumo humano necessita ser potável e não pode apresentar riscos à saúde, devendo estar ausente de microrganismos patogênicos, principalmente a Escherichia coli e os coliformes termotolerantes.

\section{Análises das condições higiênico-sanitárias do estabelecimento}

As análises das condições higiênico-sanitárias nos serviços de alimentação permitem avaliar a qualidade do ambiente e estão diretamente associadas com o percentual de adequação, dessa maneira, um total de conformidade próximo a $100 \%$ descreve uma ótima condição do estabelecimento (Neumann \& Fassina, 2016).

Segundo a coleta de informações obtidas através do checklist geral empregado, foi possível observar um baixo percentual de itens em conformidade no estabelecimento, onde o estabelecimento avaliado obteve apenas $45 \%$ de adequação, sendo classificado como grupo 3 ( 0 a 50\% de atendimento dos itens), de acordo com a RDC $n^{\circ}$ 275/2002. Isso significa que os procedimentos operacionais padrões (POP's) e as legislações em vigor no país não estão sendo seguidas rigorosamente, dando margem a possíveis contaminações de alimentos e transmissão de doenças alimentares, conforme pode ser observado na tabela 2 e gráfico 1.

Tabela 2. Distribuição do percentual (\%) de adequação das conformidades e não conformidades por cada seção do Serviço de Alimentação, segundo checklist geral.

\begin{tabular}{lll}
\hline Seções & Percentual de conformidade (\%) & Percentual de não conformidade (\%) \\
I - Edificações e instalações & $51 \%$ & $49 \%$ \\
II - Higienização & $67 \%$ & $33 \%$ \\
III - Controle integrado de vetores e & $50 \%$ & $50 \%$ \\
pragas & & $0 \%$ \\
IV - Abastecimento de água & $100 \%$ & $71 \%$ \\
V - Manejo de resíduos & $29 \%$ & \\
VI - Manipuladores & $46 \%$ & $54 \%$ \\
VII - Matérias primas, ingredientes e & $47 \%$ & $53 \%$ \\
embalagens & & \\
VIII - Preparação dos alimentos & $39 \%$ & $61 \%$ \\
IX - Armazenamento e transporte dos & $25 \%$ & $75 \%$ \\
alimentos preparados & & \\
X - Exposição ao consumo & $37 \%$ & $63 \%$ \\
XI - Documentação e registro & $0 \%$ & $100 \%$ \\
XII - Responsabilidade & $50 \%$ & $50 \%$ \\
Média final: & $45 \%$ & $55 \%$ \\
\hline
\end{tabular}

Fonte: Autores. 
Gráfico 1. Distribuição do percentual (\%) de adequação das conformidades e não conformidades por cada seção do Serviço de Alimentação, segundo checklist geral.

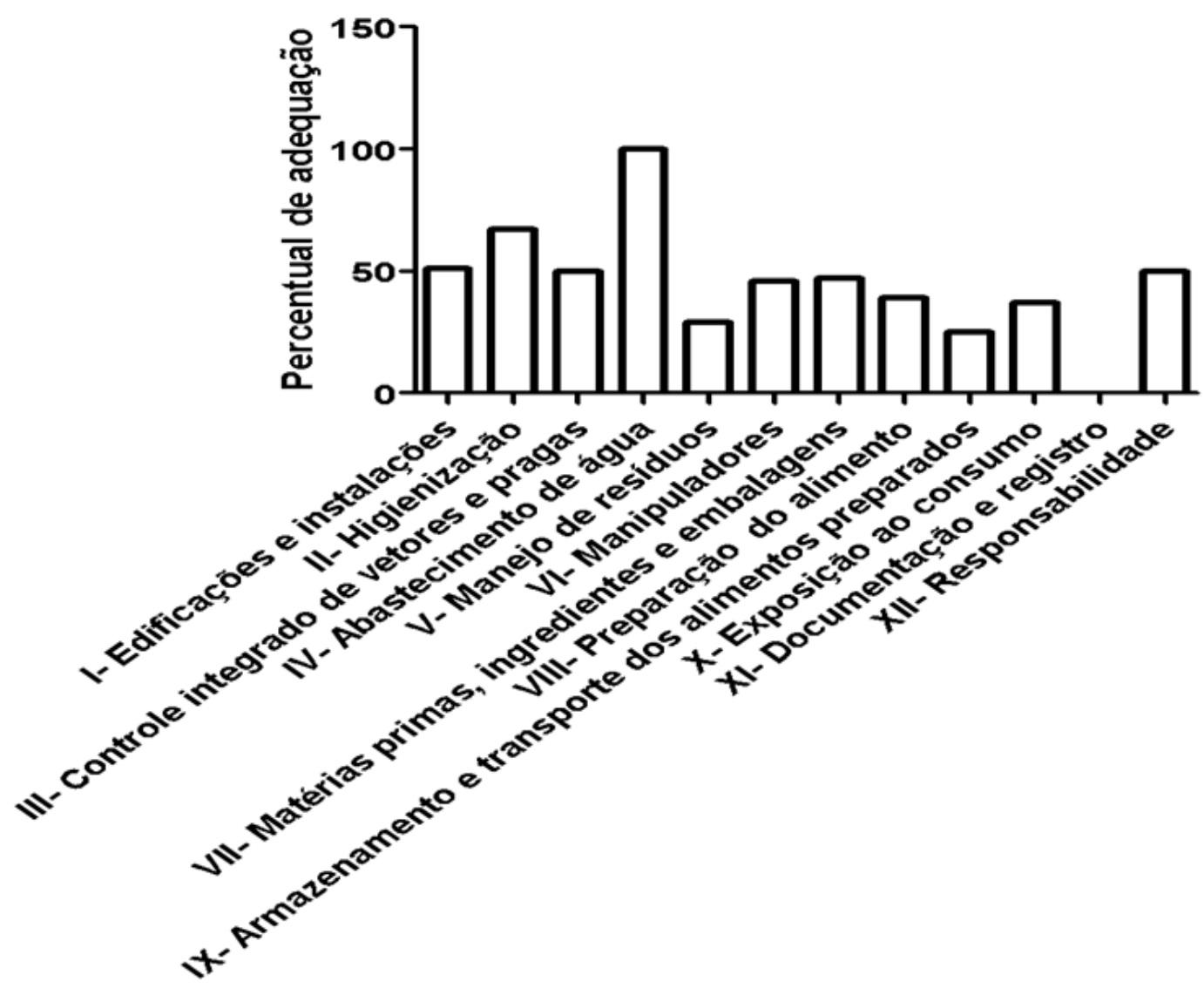

Fonte: Autores.

Na seção I, verificou-se um percentual inferior de conformidades (51\%), quando comparado com a pesquisa realizada por Lenz et al. (2019), que avaliou duas unidades de alimentação em dois municípios do Rio Grande do Sul, no qual verificouse que em uma das unidades, o percentual foi de $81,82 \%$ de conformidade na categoria edificações e instalações.

Referente à higienização da pizzaria, foi encontrado nesta pesquisa um valor de $67 \%$ de conformidades. Este valor foi superior ao observado nos estudos de Barbosa et al. (2014) a partir da avaliação de seis escolas em Itaqui/RS, no qual obtevese um desempenho insatisfatório nos itens relacionados à higiene das unidades de ensino, apresentando um percentual de conformidade de apenas 26,19\%. Dessa forma, destaca-se que o correto planejamento físico de uma UAN é importante do ponto de vista higiênico-sanitário, funcional e econômico da cozinha, pois evita que ocorra contaminação cruzada, seja ela proveniente dos manipuladores de alimentos, gêneros alimentícios, água, superfícies, equipamentos e utensílios (Texeira et al., 2007).

Na seção III, é retratado sobre o controle integrado de vetores e pragas, observando um percentual de conformidades de 50\%. Nesse contexto, é importante mencionar que após a publicação das Portarias n 326/1997 - ANVISA e n $368 / 1997$ MAPA, o controle de pragas se tornou mais taxativo, preconizando uma nova fase no desenvolvimento de práticas com todas as responsabilidades abrangentes ao controle de pragas urbanas. Também, observa-se na RDC n 52/2009 que as empresas especializadas na prestação de serviço de controle de vetores e pragas urbanas são importantes para assegurar o cumprimento das boas práticas operacionais nos estabelecimentos em que atuam. 
Referente à seção IV, relacionada ao abastecimento de água, constatou-se $100 \%$ de adequação no estabelecimento em questão; através do checklist, foi possível também avaliar que a higienização do reservatório ocorre semestralmente, atendendo aos requisitos e referências pré-estabelecidas, o que justifica o alto padrão de conformidade encontrado. Equivalentemente, no trabalho de Souza, Medeiros e Saccol (2013), o abastecimento de água apresentou 100\% de adequação às exigências da legislação.

Vale ressaltar que apesar do alto índice de conformidade, ainda é necessário analisar melhor a água, para verificação da presença de sujidades ou outros contaminantes, sendo essa diagnosticada através de laudos laboratoriais, os quais devem apresentar adequada periodicidade, e ser expedidos por empresa terceirizada e assinados por responsável técnico (Vasques \& Madrona, 2016). De acordo com a Portaria $n^{\circ}$ 2.914/2011, o responsável técnico fica encarregado pela operação do serviço de abastecimento de água, sendo obrigado a notificar a autoridade de saúde pública e informar à população em casos de descumprimento das normas.

Além disso, nesse estudo foi observada uma grande não conformidade no item $\mathrm{V}$, referente ao manejo de resíduos, indicando um percentual de $29 \%$ de conformidade. Em contrapartida, Lopes et al. (2016) em seu estudo avaliou as condições higiênico-sanitárias de um serviço de alimentação hoteleiro e constatou no mesmo item "gerenciamento de resíduos", um total de $75 \%$ de conformidade, e quanto aos itens não conformes, estes relacionam-se com a falta de coleta seletiva do lixo.

Tratando-se da seção VI, que se refere aos manipuladores, apresentou um percentual de $46 \%$ de adequação. Souza, Medeiros e Saccol (2013), por outro lado, ao analisar um serviço de alimentação em Santa Maria-RS, anteriormente à implementação do manual de boas práticas, obteve um percentual de 69\% de adequação. De acordo com Ponath (2016), a desatenção e a falta de treinamentos sobre boas práticas de manipulação, é um dos motivos que pode levar a DTAs.

Na seção VII, que trata das matérias-primas, ingredientes e embalagens, o percentual de adequação foi de $47 \%$. Este achado foi inferior ao descrito em um trabalho conduzido por Vasques e Madrona (2016), que aplicou o checklist em uma UAN, sendo ele dividido em duas etapas: a primeira foi empregada sem prévio treinamento e implantação dos manuais, e a segunda etapa ocorreu após treinamentos com a equipe, entrega do plano com ações corretivas, e implantação das Boas Práticas de Fabricação (BPF), Procedimento Operacional Padrão (POP); revelando então, uma evolução de 33\% da primeira etapa para $86 \%$ de adequações na segunda avaliação.

Oliveira (2018), através da aplicação de checklists em três estabelecimentos de Food Trucks, observou que a ausência do Manual de Boas Práticas e POP's é comum, porém relata que no primeiro Food Truck avaliado, cujo produto de venda é o açaí, houve interesse para implementação dessas documentações em seu comércio, mas ainda não aderiu por saber que o investimento é elevado e por não ter sido cobrado por nenhum órgão fiscalizador.

Quanto ao item VIII, pertinente a preparação dos alimentos, Oliveira et al. (2010) reforça a ideia das possíveis causas de DTAs, como a falta de controle quanto ao binômio tempo-temperatura, em etapas de cozimento, descongelamento, fritura e outras, cuja relevância é imprescindível na preparação alimentícia. Nesse sentido, o percentual (39\%) de conformidade obtida no estabelecimento avaliado, mostra-se aquém, e pode acarretar problemas à saúde do consumidor. Sendo assim, pode-se citar que a não higienização de alimentos crus com solução clorada é um erro rotineiramente cometido nas unidades de alimentação (Lenz et al., 2019).

$\mathrm{Na}$ categoria armazenamento e transporte dos alimentos preparados, a unidade sob estudo, apresentou um total de 25\% de conformidade, sendo considerado um dado alarmante. Neste sentido Ávila et al. (2016), relata a importância de priorizar medidas que evitem a contaminação dos alimentos, estejam eles nas etapas de preparação ou já prontos para consumo, sabendo que até que sejam entregues aos consumidores, ainda podem ser contaminados.

Em relação à exposição ao consumo dos alimentos, os resultados obtidos revelaram uma porcentagem de conformidade de apenas $37 \%$, podendo ser observadas falhas nas operações, como a falta do uso de luvas durante o preparo do 
alimento, resultando em descumprimento dos POP's e ausência na realização de exames em manipuladores de funcionários. Diferentemente, Rigui e Basso (2016), ao avaliar três instituições filantrópicas de longa permanência de idosos cadastrados pelo Programa Mesa Brasil (SESC), localizadas em Santa Maria/RS, obteve como resultado 100\% de conformidade nessa categoria. $\mathrm{O}$ que poderia ser explicado devido à natureza institucional com maior fiscalização pelos órgãos competentes.

$\mathrm{Na}$ sessão XI, referente à documentação e registro, nenhum dos itens se encontrava conforme, obtendo $0 \%$ de adequação. Esse baixo índice de conformidade se explica pela ausência do Manual de Boas Práticas de Fabricação e POP no estabelecimento. No estudo de Bernardo et al. (2014), mesmo após a implantação das BPF e dos POP foi encontrado um total de $45,2 \%$ de conformidade. É recomendado que o estabelecimento possua BPF, pois esses documentos garantem que os alimentos produzidos pela empresa estejam seguros e com qualidade sanitária aos seus consumidores (Rosa, 2015).

O item XII refere-se à responsabilidade e apresentou um percentual de 50\% de adequação, sendo observada a ausência de um responsável técnico comprovadamente capacitado em Contaminantes Alimentares, Doenças Transmitidas por Alimentos, Manipulação Higiênica dos Alimentos e Boas Práticas. Nesse contexto, Souza et al. (2021), descreve em seu estudo um percentual muito abaixo do ideal no mesmo item, apontando $0 \%$ de conformidade, relatando também a ausência de um especialista na sua unidade.

\section{Análises da gestão de desperdício}

Diante das análises realizadas na pizzaria a partir da aplicação do checklist de gerenciamento de custos, obteve-se uma média geral de adequação equivalente a $65 \%$, conforme pode ser detalhado abaixo na tabela 3 , verificou-se que o percentual de conformidade foi satisfatório, contendo 9 seções com maior porcentagem de adequação, equivalentes a uma média de $91,8 \%$, sendo eles: cardápio, compras, recebimento, preparo, porcionamento e distribuição, sobras e restos, materiais de higiene, materiais de escritório, e descartáveis. 
Tabela 3. Distribuição do percentual (\%) de adequação das conformidades e não conformidades por cada seção no Serviços de Alimentação, segundo checklist de desperdício.

\begin{tabular}{ll}
\hline Seções & Percentual de conformidade (\%) \\
I - Cardápio & $86 \%$ \\
II - Compras & $100 \%$ \\
III- Recebimento & $100 \%$ \\
IV - Armazenamento & $50 \%$ \\
V - Pré-preparo & $50 \%$ \\
VI - Preparo & $100 \%$ \\
VII - Porcionamento e distribuição & $75 \%$ \\
VIII - Fator de correção & $50 \%$ \\
IX - Sobras e restos & $67 \%$ \\
X - Mão de obra & $50 \%$ \\
XI - Tempo & $50 \%$ \\
XII - Materiais de higiene & $67 \%$ \\
XIII - Materiais de escritório & $67 \%$ \\
XIV - Descartáveis & $100 \%$ \\
XV- Eletricidade & $33 \%$ \\
XVI- Água & $05 \%$ \\
Média final: & \\
\hline
\end{tabular}

Fonte: Autores.

Por outro lado, alguns itens a exemplo de: armazenamento, pré-preparo, fator de correção, mão de obra e tempo, obtiveram uma média de $50 \%$ de porcentagens tanto para conformidades, quanto para não conformidades. É importante que essas categorias sejam bem planejadas e executadas, sendo o desperdício um indicativo importante a ser considerado durante a produção de alimentos, pois interfere diretamente na lucratividade da empresa (Schmidt, 2014).

Entre as categorias cruciais para determinar o desperdício, o fator de correção tem papel fundamental, pois é ele que determina a quantidade exata de alimento que será descartada e que deve ser empregado no planejamento quantitativo de um cardápio e consequentemente, no seu valor nutricional (Sousa, 2016). Sabe-se que a sobrevivência do Serviço de alimentação depende, em grande parte, de processos de avaliação e mensuração das perdas ocorridas, pois tais processos são de grande importância e devem ser desenvolvidos em estabelecimentos (Lemos, 2011). Resumidamente, é por meio do fator de correção que é possível prever perdas que ocorrem na etapa anterior à preparação dos alimentos. O fator de correção prevê as perdas inevitáveis durante a etapa de pré-preparo, no qual os alimentos são limpos, descascados, desossados e/ou cortados e há remoção dos talos e sementes (Ornellas, 2007). 
Os índices que apresentaram menor percentual de adequação foram os tópicos referentes à eletricidade com apenas $33 \%$ de adequação e água com nenhuma conformidade encontrada, obtendo-se um percentual de $0 \%$, obtendo uma média de 16,5\%. Segundo Rezende (2018), o desperdício está presente em todo o processo de produção de alimentos, incluindo também os indicadores de embalagens, transporte e outros. Isso reflete a necessidade de adoção de medidas sustentáveis com o intuito de economizar e aumentar o aproveitamento de recursos, como hídrico e elétrico, uma vez que estes são também fatores que contribuem bastante para os custos finais de produção (Preuss, 2009; Nunes, 2012).

Gadelha e Morais (2015) ressaltam sobre a necessidade do uso dessas ferramentas avaliativas, como checklists para manutenção do controle de qualidade do estabelecimento, considerando-se que é fundamental no gerenciamento de custos dentro em Serviços de Alimentação. Desse modo, o controle de desperdício é essencial e pode auxiliar na garantia da saúde financeira dos serviços de alimentação.

\section{Conclusão}

Este estudo permitiu demonstrar que através de uma ferramenta simples, como a aplicação da lista de verificação, foi possível avaliar as condições higiênico-sanitárias de Serviços de Alimentação e Nutrição, e a partir das observações, buscar estratégias com medidas preventivas e corretivas para o serviço em questão.

Diante disso, verificou-se a respeito da relevância do conhecimento das Boas Práticas para essas unidades, cuja condução deve ser realizada pelo nutricionista, para garantia do controle em todas as etapas como de produção e distribuição das refeições, visando à segurança dos alimentos. Além disso, a partir do planejamento administrativo do responsável técnico, é possível haver redução de custos operacionais e a otimização do tempo e espaço físico, com a utilização adequada de equipamentos, utensílios e instalações internas e de acordo com a legislação vigente. Foi possível observar também que a ausência deste profissional pode resultar em falta de gerenciamento do desperdício elevando o custo de produção dos alimentos.

A situação pandêmica exige maior cuidado e controle de qualidade dos alimentos e serviços, no entanto a problemática das boas práticas já se constitui um problema conhecido e que progride anualmente, podendo agravar ainda mais os problemas estruturais e econômicos da saúde pública. Com isso, a realização de pesquisas de cunho intervencionista em serviços de alimentação podem contribuir não somente para redução do impacto socioeconômico na pandemia, mas também para melhoria das condições de qualidade, segurança e econômica dos serviços de alimentação modificando assim realidades sociais.

\section{Referências}

Abreu, E. S., Spinelli, M. G. N., \& Pinto, A. M. S.(2003). Gestão de unidades de alimentação e nutrição: um modo de fazer. Editora Metha.

Agência Nacional de Vigilância Sanitária. (2002). Resolução $n^{\circ} 275$, de 21 de outubro de 2002. Dispõe sobre os Procedimentos Operacionais Padronizados e a lista de verificação das boas práticas de fabricação em estabelecimentos produtores/industrializadores de alimentos. Diário Oficial da República Federativa do Brasil, Brasília, DF. http://bvsms.saude.gov.br/bvs/saudelegis/anvisa/2002/anexos/anexo_res0275_21_10_2002_rep.pdf.

Agência Nacional de Vigilância Sanitária. (2004). Resolução $n^{\circ} 216$ de 15 de setembro de 2004. Dispõe sobre as boas práticas para serviço de alimentação. Diário Oficial da República Federativa do Brasil, https://bvsms.saude.gov.br/bvs/saudelegis/anvisa/2004/res0216_15_09_2004.html.

Agência Nacional de Vigilância Sanitária. (2009). Resolução $n^{\circ} 52$, de 22 de outubro de 2009. Dispõe sobre o funcionamento de empresas especializadas na prestação de serviço de controle de vetores e pragas urbanas e dá outras providências. Diário Oficial da União, Brasil. http://bvsms.saude.gov.br/bvs/saudelegis/anvisa/2009/rdc0052_22_10_2009.html.

Agência Nacional de Vigilância Sanitária. (2019). Resolução $n^{\circ}$ 60, de 23 dezembro de 2019. Estabelece as listas de padrões microbiológicos para alimentos. Diário Oficial da República Federativa do Brasil, Brasília, DF. https://www.in.gov.br/en/web/dou/-/instrucao-normativa-n-60-de-23-de-dezembro-de-2019235332356.

Almeida, V. F. D. S., Oliveira, S. R. D., Jácome, P. R. L. D. A., \& Jácome-Júnior, A. T. (2009). Assessment of physical-chemical characteristics and hygienic and sanitary conditions indicators in water supplied at public primary schools. Revista do Instituto Adolfo Lutz (Impresso), 68(3), 334-340. 
Alves, I. C., Rios, I. N. M. S., \& da Costa Matos, R. A. (2020). Avaliação do desperdício em unidade de alimentação e nutrição de instituição hospitalar pública em Brasília-DF. Brazilian Journal of Development, 6(7), 48060-48076. ). https://www.brazilianjournals.com/index.php/BRJD/article/view/13402

Ávila, M. O., Santos, P. H. S., Gois, F. N., Furtado, M. C., \& Reis, I. A. O. (2016). A importância do controle das condições microbiológicas e higiênico sanitárias na prevenção de doenças transmitidas por alimentos-uma revisão de literatura. Revista Expressão Científica, 1(1), 01-12. https://repositorio.ifs.edu.br/biblioteca/handle/123456789/766

Barboza P. P, Silveira J.T., Fagundes K. P., Pedroso Q. L. V., Brasil C. C. B., \& Garcia L. (2014). Condições higiênicas das cozinhas de escolas públicas de ensino fundamental de Itaqui-RS. Anais do Salão Internacional de Ensino, Pesquisa e Extensão, 6(2).

Bernardo, P. V., Valentim, E. C. N., de Oliveira, A. E. S., \& Ramos, S. A. (2014). Avaliação das Boas Práticas na Produção de refeições na rede Hoteleira de Belo Horizonte, MG. Journal of Health Sciences, 16(4). https://doi.org/10.17921/2447-8938.2014v16n4p\%25p

Botelho, L. V., Cardoso, L. D. O., \& Canella, D. S. (2020). COVID-19 e ambiente alimentar digital no Brasil: reflexões sobre a influência da pandemia no uso de aplicativos de delivery de comida. Cadernos de Saúde Pública, 36(11). http://dx.doi.org/10.1590/0102-311x00148020

Chaves, N. P., Bezerra, D. C., Fonseca, C. M. C., Alves, L. M. C., \& Lobato, M. S. (2014). Qualidade Microbiológica de mãos de manipuladores, equipamentos, utensílios e água de múltiplos usos em uma unidade de alimentação e nutrição na cidade de São Luís, MA. Revista Higiene Alimentar, $169-174$.

Freitas T. P, Costa T. D, Ximenes, G. R, \& Silva, C. S (2020). Condições higiênico-sanitárias de padarias de uma cidade do sertão pernambucano. In: F. Costa Barbosa. Nutrição em Foco: uma abordagem Holística. 2, 548-563. Conhecimento livre.

Gadelha, G. D. O., \& Morais, G. H. N. (2015). Análise do Processo de Desperdícios de Embalagens em uma Indústria Alimentícia: Aplicação das Quatro Primeiras Etapas do MASP. ENEGEP-Encontro Nacional de Engenharia de Produção, 35. http://www.abepro.org.br/biblioteca/TN_STO_207_228_27154.pdf

Guimarães, B. S., Ferreira, R. S., \& Soares, L. S. (2018). Perfil microbiológico de utensílios em unidade de alimentação e nutrição comercial e institucional de Salvador, BA. Revista Higiene Alimentar, 36-40. https://docs.bvsalud.org/biblioref/2018/11/965453/284-285-set-out-2018-36-40.pdf

Lemos, A. G., Botelho, R., \& Akutsu, R. D. C. C. (2011). Determinação do fator de correção das hortaliças folhosas comercializadas em Brasília. Horticultura Brasileira, 29(2), 231-236. https://www.scielo.br/pdf/hb/v29n2/a17v29n2.pdf

Lenz, B. E., Backes, J., Bertani, J. P. B., \& Fassina (2019). Verificação de boas práticas em duas unidades de alimentação e nutrição inseridas em dois municípios do Rio Grande do Sul. Revista Simbio-Logias,11(15). 62-76. https://www.ibb.unesp.br/Home/ensino/departamentos/educacao/verificacao_de_ boas_praticas_em_duas_uans.pdf

Lopes, J. E., Sousa, M. D., Chaud, D. A., Camargo, M. C. R., \& Abreu, E. S. D. (2016). Avaliação das condições higiênico sanitárias em uma unidade de alimentação e nutrição hoteleira. Higiene Alimentar, 30(256/257), 50-54. https://docs.bvsalud.org/biblioref/2016/08/1413/separata-50-54.pdf

Marques, A. S., Nespolo, C. R., R. Pinheiro, F. C., Pinheiro, F. C., \& de Magalhães Soares, G. (2017). Descontaminação microbiológica de esponjas de cozinha utilizadas em serviços de alimentação. Revista Contexto \& Saúde, 17(32), 102-114. 10.21527/2176-7114.2017.32.102-114

Ministério da Agricultura, Pecuária e Abastecimento. (1997). Portaria n. 368, de 04 de setembro de 1997. Aprova o regulamento técnico sobre as condições higiênico-sanitárias e de boas práticas de fabricação para estabelecimentos elaboradores/industrializadores de alimentos de origem animal. Brasil. https://gov.br/agricultura/pt-br/assuntos/inspecao/produtos-animal/empresario/Portaria_368.1997.pdf/view

Ministério da Saúde. (1997). Portaria n³26, de 30 de julho de 1997. Aprova o regulamento técnico sobre condições higiênico-sanitárias e de boas práticas de fabricação para estabelecimentos produtores/industrializadores de alimentos. Diário Oficial da União, Brasília, DF. https:/gov.br/agricultura/ptbr/assuntos/inspecao/produtos-vegetal/legislacao-1/biblioteca-de-normas-vinhos-e-bebidas/portaria-no-326-de-30-de-julho-de-1997.pdf/view.

Ministerio da Saúde. (2005). Portaria n518/2004. Serie E. Legislação Saúde, Ministério da saúde, Brasília. http://bvsms.saude.gov.br/bvs/publicacoes/porta ria_518_2004.pdf

Ministério da Saúde. (2013). Portaria $\operatorname{CVS} n^{\circ} 5$, de 9 de abril de 2013. Regulamenta sobre boas práticas para estabelecimentos comerciais de alimentos e para serviços de alimentação, e o roteiro de inspeção, anexo. Diário Oficial da República Federativa do Brasil, Brasília, DF. http://cvs.saude.sp.gov.br/up/PORTARIA\%20CVS-5_090413.pdf

Ministério da Saúde. Portaria $n^{\circ} 2.914$, de 12 de dezembro de 2011. Dispõe sobre os procedimentos de controle e de vigilância da qualidade da água para consumo humano e seu padrão de potabilidade. Brasil. https://bvsms.saude.gov.br/bvs/saudelegis/gm/2011/prt2914_12_12_2011.html

Monte, F.S, Santana, M.F.S, Fontes, R.S.P, Boudou, F. S. Cavalcanti, J.L.S, Silva, L.B (2021). Análise microbiológica de hortaliças em uma delicatessen na zona sul na cidade de Aracaju. Revista Eletrônica Estácio Saúde, 10(01), $7 . \quad$ http://revistaadmmade.estacio.br/ind ex.php/saudesantacatarina/article/view/6036.pdf

Neumann, L., \& Fassina, P. (2016). Verificação de boas práticas em uma unidade de alimentação e nutrição de um município do Vale do Taquari-RS. Revista Uningá Review, 26(1). http://revista.uninga.br/index.php/uningareviews/article/view/1791/1397.pdf

Nunes, R. (2010). Gastronomia Sustentável. Interação - Revista Científica da Faculdade das Américas, 3(1), $123-321$.

Oliveira, A. B. A. D., Paula, C. M. D. D., Capalonga, R., Cardoso, M. R. D. I., \& Tondo, E. C. (2010). Doenças transmitidas por alimentos, principais agentes etiológicos e aspectos gerais: uma revisão. Revista HCPA. Porto Alegre, 30(3), 279-285. http://hdl.handle.net/10183/157808.pdf

Oliveira, E. C. D. S., \& Gaveia, P. P. C. (2018). Análise de condições higiênico-sanitário dos food trucks do DF e adequação das legislações vigentes.Trabalho de Conclusão de Curso, Centro Universitário de Brasília, Brasília, Brasil. https://repositorio.uniceub.br/jspui/handle/235/12578.pdf

Ornellas, L. H. (2007). Técnica dietética: seleção e preparo de alimentos. Atheneu. 
Parisotto, D. F.; Hautrive, T. P.; \& Cembranel, M. F. (2013). Redução do desperdício de alimentos em um restaurante popular. Revista Brasileira de Tecnologia Agroindustrial, v. 7(2), 1106-1117. 10.3895/S1981-36862013000200010

Pereira, A. S., Shitsuka D. M., Parreira, F. J., \& Shitsuka, R. (2018). Metodologia da pesquisa científica [recurso eletrônico], (ed. 1, pp.1-119) Santa Maria, RS: UFSM, NTE, e-book.

Pinto, R. G. (2001). Avaliação das boas práticas de fabricação e da qualidade microbiológica na produção de pão de queijo. Dissertação de mestrado, Faculdade de Farmácia da UFMG, Belo Horizonte, Minas Gerais, Brasil.

Ponath, F. S., Valiatti, T. B., Sobral, F. D. O. S., Romão, N. F., Alves, G. M. C., \& Passoni, G. P. (2016). Avaliação da higienização das mãos de manipuladores de alimentos do Município de Ji-Paraná, Estado de Rondônia, Brasil. Revista Pan-Amazônica de Saúde, 7(1), 63-69.

Preuss, K. (2009). Integrando nutrição e desenvolvimento sustentável: atribuições e ações do nutricionista. Revista Nutrição em Pauta, 17(99), 50-53.

Rezende, C., Seemann, C. F. Silva, E. S., Jacobucci, H. B., \& Mattar, M. (2012). Superfície inanimada-possível fonte de contaminação microbiológica no alimento. Revista Brasileira de Farmácia, 93(4), 444-449.

Rezende, S. M. (2018). Estudo aplicado sobre o desperdício de alimentos em uma UAN (Unidade de Alimentação e Nutrição) de uma indústria de processamento de milho. Monografia de graduação, Universidade Federal de Uberlândia, Uberlândia, Minas Gerais, Brasil.

Rigui, K. S., \& Basso, C. (2016). Boas práticas de manipulação de alimentos em instituições filantrópicas de longa permanência de idosos. Disciplinarum Scientia| Saúde, 17(2), 279-290. https://periodicos.ufn.edu.br/index.php/disciplinarumS/article/view/2036/1882

Rosa, P. T. (2015). Implantação do manual de boas práticas de manipulação em cozinha pedagógica de uma instituição de ensino na cidade de Campo Mourão-PR. Trabalho de conclusão de curso, Universidade Tecnológica Federal do Paraná, Campo Mourão, Paraná, Brasil.

Santos, A. D. O., Sampaio, A. N. D. C. E., Martins, O. A., Pinto, J. P. D. A. N., \& Pereira, J. G. (2020). Avaliação da contaminação de equipamentos, utensílios e mãos de manipuladores de um serviço de nutrição e dietética. Archives of Veterinary Science, 25(3). http://dx.doi.org/10.5380/avs.v25i3.73104

Schmidt,V. (2014) Análise do índice de resto-ingesta em uma unidade de alimentação e nutrição do Noroeste do Estado do Rio Grande do Sul. Trabalho de Conclusão de Curso (Nutrição) - Universidade Regional de Unijuí. Unijuí, RS, Brasil.

Silva, A. N., Correia, L. M. N., Luna, J. S., Lins, J. C. (2013). Qualidade microbiológica da água utilizada em unidades de alimentação do município de Caruaru, PE. Veredas Favip-Revista Eletrônica de Ciências, 6(2), 073-082. http://blog.devrybrasil.edu.br/ojs/index.php/veredas1/article/view/65/138

Silva, F. A. M. (2019). Teoria das filas: análise do serviço em uma pizzaria. Trabalho de conclusão de curso, Universidade Federal de Campina Grande, Sumé, Paraíba, Brasil.

Sousa, M. C. (2016). Avaliação da qualidade e determinação do desperdício de vegetais durante o pré-preparo em um restaurante universitário de um campus em expansão do interior da Paraíba. Trabalho de conclusão de curso, Universidade Federal de Campina Grande, Cuité, Paraíba, Brasil.

Souza C. L \& Campos G. D. (2003). Condições higiênico-sanitárias de uma dieta hospitalar. Revista de Nutrição. 16(1):127-134.

Souza, A. O., Passos, E. V. B., Gonçalves, M. I. A., Coelho, R. L. L, Silva, C. S. (2021) Perfil higiênico-sanitário de um restaurante comercial no interior baiano. Research, Society and Development, 10 (2), 8. http://dx.doi.org/10.33448/rsd-v10i2.11870

Souza, M. D. S., Medeiros, L. B., \& Saccol, A. L. F. (2013). Implantação das boas práticas em uma unidade de alimentação e nutrição (UAN) na cidade de Santa Maria (RS). Alimentos e Nutrição Araraquara, 24(2), 208. http://serv-bib.fcfar.unesp.br/seer/index.php/alimentos/article/viewFile/2149/2149

Teixeira, S. M. F., Oliveira, Z. M. C. D., Rego, J. C. D., \& Biscontini, T. M. B. (2007). Administração aplicada às unidades de alimentação e nutrição. São Paulo (pp.81-89). São Paulo: Atheneu.

Vasques, C. T. \& Madrona, G. S. (2016). Aplicação de checklist para avaliação da implantação das boas práticas em uma unidade de alimentação e nutrição. Higiene Alimentar, 30(252/253), 54-58. https://docs.bvsalud.org/biblioref/2017/07/846570/separata-53-58.pdf

Veiga, S. M. O. M., Antonacio, N. R., \& Belmonte, M. G. (2020). Qualidade microbiológica de alimentos oriundos do comércio ambulante e intervenção educativa. Brazilian Journal of Development, 6(3), 14979-14997. https://doi.org/10.34117/bjdv6n3-393

Viana, R. M., \& Ferreira, L. C. (2017). Avaliação do desperdício de alimentos em unidade de alimentação e nutrição cidade de Januária, MG. Higiene Alimentar, 31(266/267), 22-26. https://docs.bvsalud.org/biblioref/2017/05/833306/266-267-site-22-26.pdf 